\title{
The Effects of Press Needle on Immune Function and Quality of Life Among Female Breast Cancer Patients After Radical Mastectomy: Study Protocol for a Randomised Controlled Trial
}

\section{Ying Gao}

Chengdu University of Traditional Chinese Medicine Affiliated Hospital

\section{Yao Wang}

Chengdu University of Traditional Chinese Medicine Affiliated Hospital

Hongchu Chen

Chengdu University of Traditional Chinese Medicine Affiliated Hospital

\section{Ran Yan}

Chengdu University of Traditional Chinese Medicine

Tao Liu

Chengdu University of Traditional Chinese Medicine Affiliated Hospital

Zhuohong Li ( $\square$ lizhuohong@cdutcm.edu.cn )

Chengdu University of Traditional Chinese Medicine Affiliated Hospital

Jinhao Zeng

Chengdu University of Traditional Chinese Medicine

\section{Research Article}

Keywords: breast cancer resection, immune function, press needle, study protocol

Posted Date: September 22nd, 2021

DOI: https://doi.org/10.21203/rs.3.rs-757641/v1

License: (a) (i) This work is licensed under a Creative Commons Attribution 4.0 International License. Read Full License 


\section{Abstract}

\section{Background}

Radical mastectomy may lead to suppression of cellular immune function in patients with malignant tumors, and affect the quality of life (QOL) of patients. Immune suppression is a common cause of complications and adverse reactions in adjuvant therapy after radical mastectomy of breast cancer. Currently, there are few proven effective treatments for immune suppression. Therefore, it's necessary to develop a new treatment method. Press needle is widely used in clinical practice. However, there have been relatively few studies that evaluate the effects of press needle on postoperative immune function. The aim of the present study is to assess the effects of press needle on immune function and QOL in female breast cancer patients undergoing radical mastectomy.

Methods

This study will be a single-center, randomized and single-blinded trial. 78 eligible patients will be randomized in a ratio of 1:1 to the press needle group or the sham press needle group. During the treatment phase, patients will undergo five times weekly of verum press needle or sham press needle for 2 weeks, and then they will be followed-up for 2 weeks. The primary outcome measures will be the peripheral blood levels of CD8+, CD4+, CD3+, and CD4+/CD8+ T cells. The secondary outcome measures will be the changes of patients' QOL, evaluated by the Karnofsky Performance Scale (KPS) score and the EORTC core quality of life questionnaire (EORTC QLQ-C30). Safety and adverse events will be assessed at each visit.

\section{Discussion}

The results of this on-going study will provide clinical evidence for the effects and safety of press needle on immune function and QOL in patients after breast cancer resection compared with sham press needle.

Trial registration

Chinese Clinical Trial Registry, ChiCTR2000040100. Registered on 21 November 2020.

\section{Background}

Breast cancer is one of the leading causes of death among female patients aged 35-50 years and has increasingly become a major public health burden due to its high incidence rate, high mortality rate, and tremendous health care costs ${ }^{[1-3]}$. The incidence rate of breast cancer is increasing worldwide, data from developed countries indicate that 1 in 8 women is at risk of suffering from breast cancer ${ }^{[4,5]}$. China has the largest breast cancer population in the world, and the annual increasing rate is about $1-2 \%$ higher than the world average $\mathrm{e}^{[6]}$. 
Radical surgery is currently the preferred treatment for breast cancer, and about $70-80 \%$ of patients with early non-metastatic disease can be cured ${ }^{[7]}$. Postoperative adjuvant therapy can reduce the recurrence rate of breast cancer and prolong the survival of patients, which is of great significance in the treatment of breast cancer ${ }^{[8,9]}$. Although some progress has been made in the comprehensive treatment, recurrence and metastasis are still important factors affecting patients' prognosis ${ }^{[10]}$. Studies have shown that lymphocytes, including $T$ cell and natural killer (NK) cells, are related to the primary prevention and recurrence of breast cancer ${ }^{[11]}$. Specially, $T$ cell-mediated immune response can kill circulating tumor cells and micro-metastatic lesions to prevent tumor recurrence and metastasis ${ }^{[12]}$. However, surgery-related neuroendocrine stress responses, blood transfusion, anesthetics, and opioid analgesics are factors known to adversely affect the anti-tumor immune defenses ${ }^{[13]}$. The immunosuppressive state after surgery may lead to poor adjuvant treatment effect, and patients are prone to develop complications and adverse reactions, including anxiety, cancer-related insomnia, postoperative infection, radiation dermatitis, and even grade IV bone marrow suppression ${ }^{[14]}$. This affects the quality of life (QOL) of patients. Thus, it is important to improve the immune function for postoperative patients with breast cancer.

At present, no therapy is effective at improving immune suppression. Due to the limited efficacy and many side effects of drug therapy, various non-pharmacological techniques have been used in clinical practice $^{[15]}$. A large number of clinical studies have shown that acupuncture has a definite effect on enhancing immunodeficiency and improving the side effects of adjuvant therapy after radical mastectomy of breast cancer ${ }^{[16-18]}$, and it also shows significant advantages in the treatment of chronic urticarial, inflammatory bowel disease and other types of immunodeficiency diseases ${ }^{[19-21]}$. The intradermal thumbtack needle, an improved subcutaneous needle, is a type of shallow needling method that reduces pain and prolongs the acupuncture effect via longer needle retention ${ }^{[22]}$. It has the characteristics of long-lasting efficacy, safety and convenience. Currently, only a small number of studies have evaluated the effects of press needle on the treatment of immunodeficiency after breast cancer resection. Therefore, the purpose of this study is to evaluate the effects and safety of press needle on immune function in postoperative patients with breast cancer.

\section{Objectives}

The objectives of the study are:

1. To investigate the effectiveness of press needle of immunocompromised patients after breast cancer resection, when compared with sham press needle.

2. To compare the differences in improvement of QOL, measured by the KPS score and the EORTC QLQC30 score, between the intervention group and control group.

3. To assess the safety of press needle treatment, all adverse events will also be evaluated. 


\section{Methods/design}

Study design and ethics

This is a single-center, randomized, sham-controlled clinical trial. The trial will be conducted at Affiliated Hospital of Chengdu University of Traditional Chinese Medicine after ethical approval has been obtained from the Institutional Review Board (Ethical Approval Number: 2021KL-049). Written informed consent will be obtained from all study participants prior to enrollment. Eligible patients will be randomly divided into the press needle group and the sham press needle group in a 1:1 allocation ratio. Participants will be evaluated and results will be analyzed by professionals blinded to group allocation. The flow chart of the study process is shown in Figure 1.

Sample size

According to the literature study ${ }^{[23]}$, the mean effect and standard deviation of CD3+ value after radical breast cancer surgery were 57.74 and 7.74, respectively. After acupuncture intervention, the mean effect of $C D 3+$ value is estimated to be 52.37 , and the standard deviation is about 6.87 , expecting that the effect of press needle is similar to that of intervention. Pass 15.0 software was used to calculate the sample size of 31 in each group and 62 patients in total under the condition that the ratio between groups was $1: 1$, the significance level of bilateral test a was 0.05 , and the degree of assurance (1- $\beta$ ) was 0.80 . Considering the $20 \%$ dropout rate, the total sample size was 78 patients, and 39 in each group.

\section{Participants}

\section{Inclusion criteria}

Participants with the following conditions will be included:

1. Patients who have undergone radical mastectomy.

2. Did not receive radiotherapy, chemotherapy or any other drugs that affect immune function before hospitalization.

3. No other tumors, acute or chronic inflammations, blood system diseases and autoimmune diseases.

4.Age $18-80$ years, female gender.

5. Eastern Cooperative Oncology Group (ECOG) performance status (PS) of 0 to 2.

6. Life expectancy $\geq 3$ months.

7. With normal bone marrow, heart, liver and kidney functions.

8. Ability to understand and the willingness to sign a written informed consent document. 
9. Capable of providing responses during outcome measurement.

\section{Exclusion criteria}

Participants with the following conditions will be excluded:

1. History of other malignant tumors within 5 years.

2. Severe cardiac or respiratory diseases, significant renal or hepatic impairment or immune disorders.

3. Active infection, including active tuberculosis infection.

4. Severe infection after surgery.

5. History of immunosuppressive therapy (chemotherapy or radiation) or previous acupuncture/press needle therapies.

6. Life expectancy $₫ 3$ months.

7. Pregnancy or currently lactating.

8. Severe mental disorders such as cognitive impairment.

9. Refusal to accept press needle.

10. Participating in other clinical trials.

Randomization and allocation concealment

A total of 78 participants who meet the eligibility criteria will be randomized in a ratio of $1: 1$ to the press needle or sham press needle group. An independent statistician will generate the randomization sequence using SPSS 22.0. The randomization procedure will be conducted by a research assistant who will not take part in the interview, data collection, intervention, or statistical analysis. The randomization will be concealed using opaque envelopes. Informed consent and baseline data collection will be completed prior to randomization. Sequentially numbered opaque sealed envelopes containing the randomization assignments will be delivered to the clinical trial centers. Allocation concealment will not be broken until the final data analysis report is completed.

Blinding

In this study, doctors will know the allocation of each patient, so it is not feasible to conceal the allocation from doctors. However, treatment and assessment will be performed independently, doctors will not be involved in evaluating the outcome of treatment. The outcome assessors and the statistician performing the data analyses will be blinded to treatment allocation throughout the study.

Intervention 


\section{The press needle group}

Press needle will be administered five times weekly for two weeks. According to our previous experience, Zhongwan (CV12), Geshu(BL17), Pishu(BL20), bilateral Hegu (LI4), bilateral Sanyinjiao (SP6) and bilateral Zusanli (ST36) will be used as the acupoints for treatment. The location of the acupoints willrefer to national standards of PRC published in 2006 (GB/T 12 346-2006) ('The Name and Positioning of Acupoints') ${ }^{[24]}$. After skin cleansing with a $75 \%$ alcohol swab, Japanese Seirin Pyonex $(0.2 \times 1.5 \mathrm{~mm})$ will be quickly inserted into the skin and embedded at the selected acupoints. Instruct the patient to press for one minute at each acupoint once every 4 hours, with slight pain and soreness as appropriate. The needle will be replaced after 24 hours. In case of fainting and skin allergy during acupuncture, remove the needle in time.

\section{The control group}

The control group will undergo treatment with special sham press needle. We will use a non-invasive placebo control in which the needle element of the snap is removed and the skin around each acupoint is touched under the same pressure. Patients will feel touch, but no pain or heaviness. Positional acupoint selection and intervention time will be the same as the treatment group. Sham treatment will be performed by the same doctors who perform the verum treatment.

\section{Prohibited concomitant treatments}

In both groups, all additional treatments for immunodeficiency, including drugs, supplements, and alternative treatments, will be prohibited during the study period.

Outcome assessment

We will assess the primary outcome at baseline, week 1 and week 2 of treatment, and at follow up at the 3rd and 4th weeks after the end of treatment. Secondary outcomes will be assessed at baseline, week 2 and week 4.

\section{Primary outcome measurements}

The primary outcome will be the changes in peripheral blood T lymphocyte subsets $\left(\mathrm{CD} 8^{+}, \mathrm{CD} 4^{+}, \mathrm{CD} 3^{+}\right.$, and $\mathrm{CD} 4^{+} / \mathrm{CD} 8^{+} \mathrm{T}$-cell ratio).

\section{Secondary outcome measurements}

The secondary outcomes will include the KPS score and the EORTC QLQ-C30 score to assess the patient's quality of life.

The schedule of procedures and evaluations is presented in Table 1.

Quality control 
The Clinical Research Center of Drugs of Chengdu University of Traditional Chinese Medicine Data Monitoring Team will be responsible for controlling bias and identifying problems in the project. In order to guarantee the quality of this study, every acupuncturist in this trial will be a registered practitioner with 3-5 years of clinical experience in the practice of acupuncture. Meanwhile, a qualified clinical trial expert will monitor every trial center, and regular board meetings will be held to ensure the quality of the study process.

Statistical analysis

SPSS 22.0 statistical software will be used for data analysis, and an $\chi 2$ test will be used for counting data. Data are expressed as $x \pm s$. If the assumptions of normal distribution and homogeneity of variance are met, a t-test will be used to determine differences; otherwise, nonparametric test will be employed. A $P<0.05$ is considered to be statistically significant.

Safety and monitoring

All adverse events will be observed and reported by patients and researchers during each patient visit. Any expected or unexpected adverse events related to this study will be recorded and monitored until its resolution. Safety assessment will be performed at the screening visit and after the end of treatment, including blood routine, urine routine, stool routine, liver and kidney function, coagulation function, and electrocardiogram. In addition, at each visit, vital signs will be measured, and adverse events will be recorded.

Data and safety monitoring will be conducted at periodic intervals during the study. The monitors will check study protocol compliance and informed consent documents and evaluate the progress of the trial, including participant recruitment, data quality and timeliness.

Table 1 Timing of treatment assessments and data collection 


\begin{tabular}{|c|c|c|c|c|c|c|}
\hline Period & Enrollment & Baseline & Treatment phase & \multicolumn{3}{|c|}{ Follow-up phase } \\
\hline Week & -1 & 0 & 1 & 2 & 3 & 4 \\
\hline \multicolumn{7}{|l|}{ Enrollment } \\
\hline Eligibility screen & $x$ & & & & & \\
\hline Informed consent & $x$ & & & & & \\
\hline Medical history & $x$ & & & & & \\
\hline Allocation & & $x$ & & & & \\
\hline \multicolumn{7}{|l|}{ Interventions } \\
\hline Press needle & & & $x$ & $x$ & & \\
\hline Sham press needle & & & $x$ & $x$ & & \\
\hline \multicolumn{7}{|l|}{ Assessments } \\
\hline \multicolumn{7}{|l|}{ Primary outcome } \\
\hline T-cell ratio & & $x$ & $x$ & $x$ & $x$ & $x$ \\
\hline \multicolumn{7}{|l|}{ Secondary outcomes } \\
\hline KPS & & $x$ & & $x$ & & $x$ \\
\hline EORTC QLQ-C30 & & $x$ & & $x$ & & $x$ \\
\hline Safety assessment & & $x$ & $x$ & $x$ & $x$ & $x$ \\
\hline
\end{tabular}

KPS Karnofsky Performance Scale, EORTC QLQ-C30 European Organization for Research and Treatment of Cancer Quality of Life Questionnaire

\section{Discussion}

Surgery-related neuroendocrine stress responses, blood transfusion, anesthetics, and opioid analgesics are factors known to adversely affect the anti-tumor immune defenses ${ }^{[13]}$. The immunosuppressive state after surgery may lead to poor adjuvant treatment effect, and patients are prone to complications and adverse reactions ${ }^{[25]}$, thereby affecting their quality of life. With the development of complementary and alternative medicine, more and more patients with cancer tend to choose acupuncture as a main treatment to improve immunodeficiency. Recently, several studies have shown that acupuncture can effectively improve immunodeficiency ${ }^{[26-29]}$. The intradermal thumbtack needle, an improved subcutaneous needle, is a type of shallow needling method that reduces pain and prolongs the acupuncture effect via longer needle retention ${ }^{[22]}$. It has the characteristics of long-lasting efficacy, safety and convenience. However, only a small number of studies have evaluated the effects of press needle on 
the treatment of immunodeficiency after breast cancer resection. In this study protocol, we designed a randomized, sham-controlled, single-blinded trial.

Our study protocol is based on literature from textbooks and relevant reports, and on our previous clinical experience. Traditional acupoints (CV12, BL17, BL20 and bilateral LI4, SP6, and ST36) will be selected for treatment. The press needle used in this study is easy to apply, it can be applied anywhere or anytime and can be effective for a long duration without serious complications when compared to electronic acupuncture that only works for a short time ${ }^{[30]}$. The experimental group was treated with press needle, while the control group received sham press needle treatment. Treatment was performed five consecutive times a week with 2 days of rest for 2 weeks. In this study, peripheral blood T lymphocyte subsets (CD ${ }^{+}$, $\mathrm{CD}^{+}, \mathrm{CD}^{+}$, and $\mathrm{CD} 4^{+} / \mathrm{CD}^{+}$T-cell ratio) will be administered to evaluate the immune function. The KPS score and the EORTC QLQ-C30 score to assess the patient's quality of life. This study can contribute to a better understand changes in immune function or quality of life in patients after breast cancer surgery.

However, this study protocol still faces several limitations and challenges. The first is the blinding method. Because of the nature of the clinical trials of acupuncture, it is inevitable that the acupuncturist will know the treatment allocation. However, the acupuncturists will be blinded to the results. In order to prevent the acupuncturist from accidentally revealing the group allocation, their interactions with the patients will be limited. Overall, both groups will receive blinding to balance the efficacy between the two groups. The second limitation is the application of the acupuncture method. Before the study begins, all acupuncturists will receive several training sessions on the standardized protocol for point selection and needle insertion to ensure the consistency of the acupuncture techniques. After the trial is completed, researchers will conduct follow up by phone to collect data on patients' final outcomes.

Though there are many challenges, we will strive to standardize the steps of the trial and the quality of the trial will be monitored by the Data and Safety Monitoring Board (DSMB). We hope that this trial will provide a strong quality of evidence on the efficacy and safety of press needle for improving immune function and QOL.

\section{Trial status}

This trial is currently recruiting participants.

\section{Abbreviations}

ECOG PS: Eastern Cooperative Oncology Group Performance Status; EORTC QLQ-C30: EORTC core quality of life questionnaire; QOL: Quality of life; RCT: randomized controlled trial; DSMB: Data and Safety Monitoring Board.

\section{Declarations}


We would like to thank Dr Zhuohong Li from the oncology department, Affiliated Hospital of Chengdu University of Traditional Chinese Medicine, for her language editorial support.

\section{Funding}

This research was supported by the Fund of Affiliated Hospital of Chengdu University of Traditional Chinese Medicine(grant number: 20ZL15) and“Hundred Talents Program” of Affiliated Hospital of Chengdu University of Traditional Chinese Medicine (grant number: 20-Q17).

\section{Authors' contributions}

The trial was designed and developed by YG and YW. HCC provided statistical support. RY, TL contributed to the design of the intervention. The manuscript was prepared by YG. The protocol was carefully revised and edited by $\mathrm{ZHL}$ and $\mathrm{JHZ}$. All authors read and approved the final manuscript.

\section{Ethics approval and consent to participate}

The protocol of this study has been reviewed and approved by the Institutional Review Board of Human Research of Affiliated Hospital of Chengdu University of Traditional Chinese Medicine (2021 KL-049). All participants will give their written informed consent to the research assistant before joining this study.

\section{Competing interests}

The authors declare that they have no competing interests.

\section{Author details}

1 Affiliated Hospital of Chengdu University of Traditional Chinese Medicine, Chengdu, 610072, China

2 Chengdu University of Traditional Chinese Medicine, Chengdu, 610075, China.

\section{References}

[1].Rojask, Stuckeya. Breast cancer epidemiology and risk factors[J]. Clin Obstet Gynecol, 2016,59(4):651672.

[2].Furrukh M, Qureshi A. Treatment of breast cancer; review and updates[J]. J Ayub Med Coll Abbottabad, 2018,30(2):264-274.

[3].Li N, Deng Y, Zhou L, et al. Global burden of breast cancer and attributable risk factors in 195 countries and territories, from 1990 to 2017: results from the Global Burden of Disease Study 2017[J]. J Hematol Oncol, 2019,12(1):140.

[4].Coughlin SS. Epidemiology of breast cancer in women[J]. Adv Exp Med Biol, 2019;1152:9-29. 
[5].Winters S, Martin C, Murphy D, et al. Breast cancer epidemiology, prevention, and screening[J]. Prog Mol Biol Trans/ Sci, 2017;151:1-32.

[6].Ghoncheh Mahshid, Momenimovahed Zohre, Salehiniya Hamid. Epide-miology, incidence and mortality of breast cancer in asia[J]. Asian Pac J Cancer Prev, 2016,17(S3):47-52.

[7].Harbeck N, Frédérique Penault-Llorca, Cortes J, et al. Breast cancer[J]. Nat Rev Dis Primers, 2019,5(1):66.

[8].Abdel-Rahman Omar. Impact of timeliness of adjuvant chemotherapy and radiotherapy on the outcomes of breast cancer; a pooled analysis of three clinical trials[J]. Breast, 2018,38:175-180.

[9].Abram Recht, Elizabeth A Comen, Richard E Fine, et al. Postmastectomy Radiotherapy: An American Society of Clinical Oncology, American Society for Radiation Oncology, and Society of Surgical Oncology Focused Guideline Update[J]. J Clin Oncol, 2016,6(6):e219-e234.

[10].Valachis A, Mamounas E P, Mittendorf E A, et al. Risk factors for locoregional disease recurrence after breast-conserving therapy in patients with breast cancer treated with neoadjuvant chemotherapy: An international collaboration and individual patient meta-analysis: Recurrence After Neoadjuvant Chemotherapy[J]. Cancer, 2018,124(14):2923-2930..

[11].Standish L J, Sweet E S, Novack J, et al. Breast cancer and the immune system[J]. J Soc Integr Oncol, 2008,6(4):158-168.

[12].Sun Y-F, Yang X-R, Zhou J, et al. Circulating tumor cells: Advances in detection methods, biological issues, and clinical relevance[J]. J Cancer Res Clin Oncol, 2011,137(8):1151-1173.

[13].Chen Z, Zhang P, Xu Y, et al. Surgical stress and cancer progression: the twisted tango[J]. Mol Cancer, 2019,18(1):132.

[14].Elliott, RL, Jiang, et al. Human leukocyte antigen $\mathrm{G}$ expression in breast cancer: role in immunosuppression[J]. Cancer Biother Radiopharm, 2011,26(2):153-157.

[15].Martins F, Sofiya L, Sykiotis G P, et al. Adverse effects of immune-checkpoint inhibitors: epidemiology, management and surveillance[J]. Nat Rev Clin Oncol, 2019,16(9):563-580.

[16].Yuanqing P, Yong T, Haiqian L, et al. Acupuncture for Hormone Therapy-Related Side Effects in Breast Cancer Patients: A GRADE-Assessed Systematic Review and Updated Meta-Analysis[J]. Integr Cancer Ther, 2020,19:153473542094039.

[17].Nahleh Z, Tabbara I A. Complementary and alternative medicine in breast cancer patients[J]. Palliat Support Care, 2003,1(3):267-273. 
[18].Tagliaferri M, Cohen I, Tripathy D. Complementary and alternative medicine in early-stage breast cancer[J]. Semin Oncol, 2001,28(1):121-134.

[19].Shi Y, Zheng H, Zhou S, et al. Efficacy and safety of acupuncture for patients with chronic urticaria: study protocol of a randomized, sham-controlled pilot trial[J]. Trials, 2019,20(1):326.

[20].Song G, Fiocchi C, Achkar JP. Acupuncture in Inflammatory Bowel Disease[J]. Inflamm Bowel Dis, 2019,25(7):1129-1139.

[21].Da Silva MD, Bobinski F, Sato KL, et al. IL-10 Cytokine Released from M2 Macrophages Is Crucial for Analgesic and Anti-inflammatory Effects of Acupuncture in a Model of Inflammatory Muscle Pain[J]. Mol Neurobiol, 2015,51(1):19-31.

[22].Jian-qin Lv, Chengwei Wang, Yi Yang, et al. Intradermal thumbtack needle buried Neiguan (P6) point for prevention of postoperative nausea and vomiting in patients undergoing craniotomy: study protocol for a randomised controlled trial[J]. BMJ Open, 2019,9(11):e032417.

[23].SUN Ning, ZHOU Min-tao. Analgesic Effect of Acupuncture After Surgery for Breast Cancer and Its Influence on TNF-a, IL-6 and Immune Function [J]. Shanghai J Acu-mox, 2019,38(12):1350-1354.

[24].Huang Long-xiang, Zhao Jing-sheng, Wu Zhong-chao, et al. Editorial explanation on the state standard The Name and Location of Acupoints(2006, edition)[J]. Chinese acupuncture \& moxibustion, 2009, 29(11):924-926.

[25].Sherry A D, Eyben R V, Ms N B N, et al. Systemic Inflammation After Radiation Predicts Locoregional Recurrence, Progression, and Mortality in Stage II-III Triple-Negative Breast Cancer[J]. Int J Radiat Oncol Biol Phys, 2020,108(1):268-276.

[26].Roosterman D, Goerge T, Schneider SW, et al. Neuronal Control of Skin Function: The Skin as a Neuroimmunoendocrine Organ[J]. Physiol Rev, 2006,86(4):1309-1379.

[27].Takano T, Chen X, Luo F, et al. Traditional Acupuncture Triggers a Local Increase in Adenosine in Human Subjects[J]. J Pain, 2012,13(12):1215-1223.

[28].Yao W, Yang H, Yin N, et al. Mast Cell-Nerve Cell Interaction at Acupoint: Modeling Mechanotransduction Pathway Induced by Acupuncture[J]. Int J Biol Sci, 2014,10(5):511-519.

[29].Pan W X, Fan A Y, Chen S, et al. Acupuncture modulates immunity in sepsis: Toward a science-based protocol[J]. Auton Neurosci, 2021,232(15-41):102793.

[30].Ruengwongroj P, Muengtaw Ee Pongsa S, Patumanond J, et al. Effectiveness of press needle treatment and electroacupuncture in patients with postherpetic neuralgia: A matched propensity score analysis[J]. Complement Ther Clin Pract, 2020:101202. 


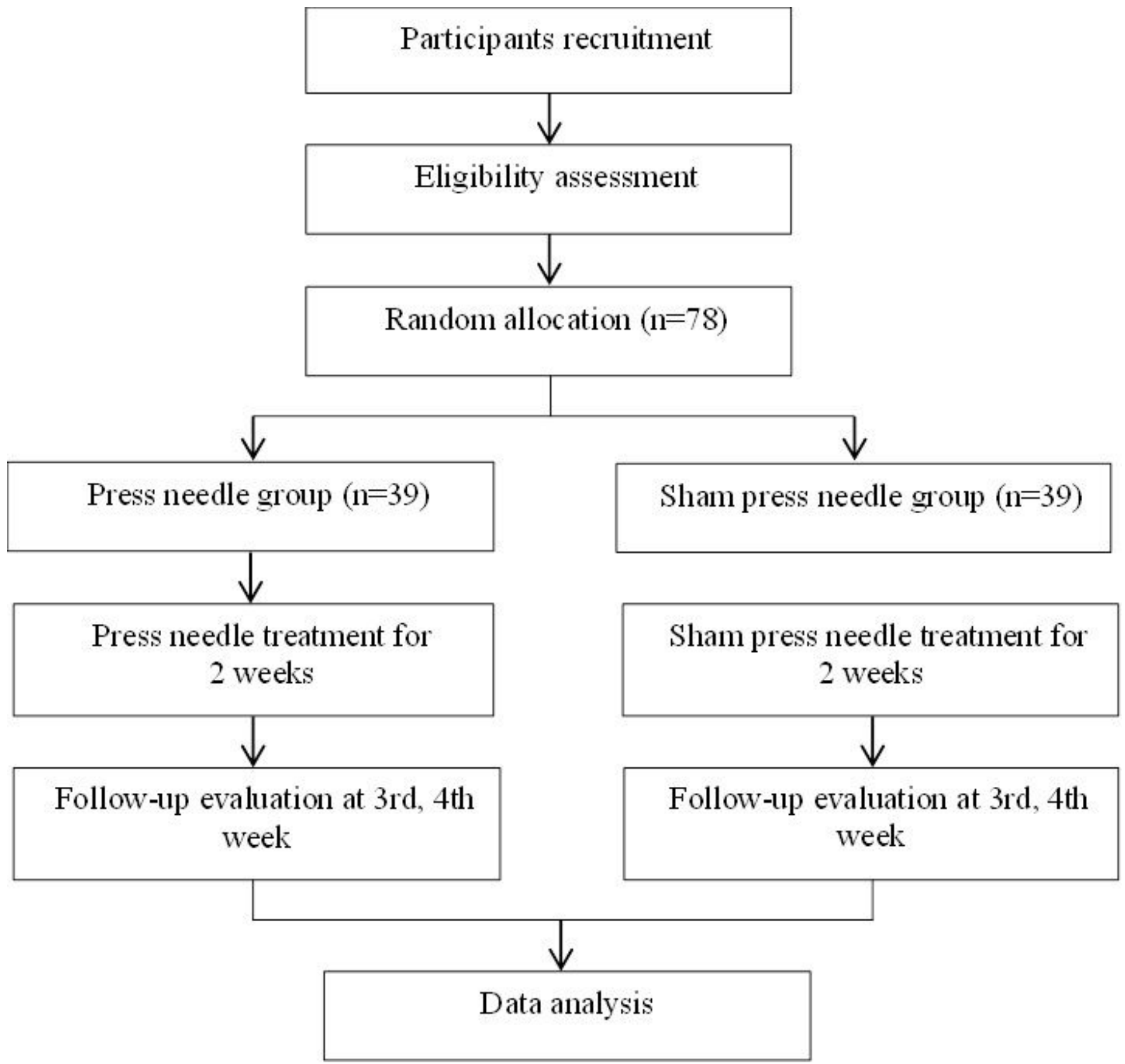

Figure 1

Flowchart showing the steps in patient recruitment, treatment, and analysis.

\section{Supplementary Files}

This is a list of supplementary files associated with this preprint. Click to download.

- Informedconsentform.docx 
- Theeffectsofpressneedleonimmunefunction.pdf 